\title{
Using Evoked Potentials to Match Interaural Electrode Pairs with Bilateral Cochlear Implants
}

\author{
Zachary M. Smith ${ }^{1,2,4}$ and Bertrand Delgutte ${ }^{1,2,3}$ \\ ${ }^{1}$ Eaton-Peabody Laboratory, Massachusetts Eye and Ear Infirmary, Boston, MA, USA \\ ${ }^{2}$ Speech and Hearing Bioscience and Technology Program, Harvard-MIT Division of Health Sciences and Technology, \\ Cambridge, MA, USA \\ ${ }^{3}$ Research Laboratory of Electronics, Massachusetts Institute of Technology, Cambridge, MA, USA \\ ${ }^{4}$ Department of Biomedical Engineering, Johns Hopkins University School of Medicine, Baltimore, MD, USA
}

Received: 18 September 2006; Accepted: 10 December 2006; Online publication: 17 January 2007

\begin{abstract}
Bilateral cochlear implantation seeks to restore the advantages of binaural hearing to the profoundly deaf by providing binaural cues normally important for accurate sound localization and speech reception in noise. Psychophysical observations suggest that a key issue for the implementation of a successful binaural prosthesis is the ability to match the cochlear positions of stimulation channels in each ear. We used a cat model of bilateral cochlear implants with eight-electrode arrays implanted in each cochlea to develop and test a noninvasive method based on evoked potentials for matching interaural electrodes. The arrays allowed the cochlear location of stimulation to be independently varied in each ear. The binaural interaction component (BIC) of the electrically evoked auditory brainstem response (EABR) was used as an assay of binaural processing. BIC amplitude peaked for interaural electrode pairs at the same relative cochlear position and dropped with increasing cochlear separation in either direction. To test the hypothesis that BIC amplitude peaks when electrodes from the two sides activate maximally overlapping neural populations, we measured multiunit neural activity along the tonotopic gradient of the inferior colliculus (IC)
\end{abstract}

Correspondence to: Zachary M. Smith • Department of Biomedical Engineering • Johns Hopkins University School of Medicine • Baltimore, MD, USA. Telephone: +1-410-9553162; fax: +1-4109551299; email: zsmith@ jhu.edu with 16-channel recording probes and determined the spatial pattern of IC activation for each stimulating electrode. We found that the interaural electrode pairings that produced the best aligned IC activation patterns were also those that yielded maximum BIC amplitude. These results suggest that EABR measurements may provide a method for assigning frequencychannel mappings in bilateral implant recipients, such as pediatric patients, for which psychophysical measures of pitch ranking or binaural fusion are unavailable.

Keywords: binaural hearing, electric stimulation, neural prosthesis, cochlear implant, inferior colliculus

\section{INTRODUCTION}

Cochlear prostheses are increasingly being implanted in both ears in the hope of improving hearing through the implants by taking advantage of binaural cues important in normal hearing individuals for sound localization and speech reception in noise (Durlach and Colburn 1978; Zurek 1993; Blauert 1997). Beyond traditional binaural advantages based on binaural difference cues, which require the comparison of timing and intensity information across the ears, and the head shadow effect, which reflects the ability to attend to the ear with the most favorable target-to-masker ratio, some bilateral cochlear implant users have better speech reception with the addition of redundant or complementary 
speech information across the ears (Schon et al. 2002; Long et al. 2004; Litovsky et al. 2006). This effect, often referred to as binaural summation, further highlights the ability of the auditory system to neurally combine information across the ears.

A hallmark of the auditory system is the tonotopic organization that is established in the cochlea and maintained through its ascending pathways to the primary auditory cortex. This organization is also maintained in the early connections between auditory neurons from the two sides of the head. In the medial superior olive (Guinan et al. 1972; Yin and Chan 1990), the lateral superior olive (Boudreau and Tsuchitani 1968), and the inferior colliculus (IC) (Kuwada et al. 1984; Qiu et al. 2003), binaural neurons have very similar characteristic frequencies (CFs) for monaural stimulation of the two ears. Bilateral cochlear implants must correctly assign frequency channels along the electrode arrays in each ear such that bilateral information is properly aligned once it converges on the binaural neurons of the auditory CNS. Such an alignment may be important for both binaural hearing and the fusion of speech information across ears.

Potential benefits of having closely matched frequency-place assignments across the ears include improved speech reception and better sound localization. It has been shown that shifting or warping of the natural frequency-place map is detrimental to speech reception with both unilateral cochlear implants (Baskent and Shannon 2004) and simulations of cochlear implants in normal-hearing subjects (Dorman et al. 1997; Shannon et al. 1998; Baskent and Shannon 2003). While subjects can often adapt to shifts in the frequency-place map over time (Rosen et al. 1999), simulations of bilateral implants in normal-hearing subjects (Siciliano et al. 2006) suggest that an offset between frequency-place maps in the two ears is not only detrimental to speech reception but also difficult to adapt to. Behavioral results in a bilaterally implanted human subject suggest that sensitivity to interaural time differences (ITDs) is best for interaural electrode pairs that excite the same tonotopic region of the auditory nerve (Long et al. 2003). Similarly, acoustic sensitivity to ITD in the envelope of high-frequency, sinusoidally amplitude-modulated tones is also best when the carrier frequencies, and therefore the cochlear locations of stimulation, are the same in the two ears (Nuetzel and Hafter 1981). In normal-hearing listeners, binaural improvements for speech reception in noise depend partly on differences in ITD between the target and the masker (Zurek 1993). It is likely that these improvements also require a frequencyaligned convergence of information between the two ears.
One of the first studies of a bilaterally implanted patient (Pelizzone et al. 1990) showed binaural interactions in the brainstem-evoked response with binaural electric stimulation of the cochlea. Pelizzone and colleagues hypothesized that the binaural interaction component (BIC) of the electrically evoked auditory brainstem response (EABR) should be greatest when the two implants stimulate auditory nerve fibers innervating comparable regions in the two cochleae. They proposed that this could provide a way to symmetrically position cochlear implants in each ear. This paper develops and investigates this idea by measuring evoked potentials with stimulation through different electrode pairs in bilaterally implanted cats and testing whether BIC amplitude is greatest for matched interaural electrode pairs. This noninvasive method could in principle work in humans, so it has potential clinical usefulness. We also used an invasive measure of neural activity along the tonotopic axis of the IC to more directly identify the neural populations excited by each individual stimulus electrode and make interaural electrode assignments based on the alignment of the activated populations. This direct method is used to test the results of the noninvasive evoked potential method.

\section{METHODS}

\section{Subjects and deafening}

All surgical and experimental procedures followed the regulations set by NIH and were approved by the internal animal care and use committees of both the Massachusetts Eye and Ear Infirmary and the Massachusetts Institute of Technology. Healthy adult cats of either sex were deafened by coadministration of kanamycin (300 mg/kg subcutaneous) and ethacrynic acid ( $25 \mathrm{mg} / \mathrm{kg}$ intravenous) 7-14 days before cochlear implantation and electrophysiological recordings (Xu et al. 1993).

\section{Surgery}

On the day of the experiment, after induction of anesthesia by Dial in urethane $(75 \mathrm{mg} / \mathrm{kg})$, a tracheal canula was inserted; skin and muscles overlying the back and top of the skull were reflected. Ear canals were transected for insertion of a calibrated closed acoustic system. Tympanic bullae were opened to allow access to the round window for insertion of intracochlear electrodes. Part of the skull overlying the occipital cortex was removed to allow for partial aspiration of cortical tissue and access to the bony tentorium and IC. Part of the tentorium overlying the IC was drilled for better access to the dorsolateral surface of the IC. Throughout all procedures, animals 
were given supplementary doses of anesthesia to maintain an areflexic state, and vital signs were monitored.

\section{Cochlear implantation and electrode configurations}

Stimulating electrode arrays were surgically implanted into each cochlea through the exposed round window. The arrays (Cochlear Corp.) had eight ring-type contacts with $0.45 \mathrm{~mm}$ diameter and $0.75 \mathrm{~mm}$ spacing between contacts. The contacts were numbered 18 from apical to basal. Particular care was taken to achieve the same insertion depth on both sides as observed by an operating microscope. The depth of insertion was 5-6 $\mathrm{mm}$ depending on the animal, and the most basal contact (\#8) was typically just at the round window. Absorbent cotton spears were placed lateral to each electrode array and used to push the array against the modiolus.

Several intracochlear electrode configurations were used, including monopolar (MP), bipolar $(\mathrm{BP})$, and bipolar plus one $(\mathrm{BP}+1)$ configurations. This choice of configurations reflects those that are used in clinical devices. For all configurations, current was passed between an active electrode and a return electrode. The active electrode was always one of the electrodes on the implanted array, and the only difference between electrode configurations is the location of the return electrode. For monopolar configuration, the return electrode was a needle inserted in the neck muscle. For bipolar configuration, the return electrode was always the intracochlear electrode just one contact basal of the active electrode. Bipolar plus one is similar to the bipolar configuration except that the return electrode is two contacts basal of the active electrode. Although there was always a pair of electrodes for each configuration in each ear, only the position of the active electrode (the most apical of the pair) is reported because the location of the return electrode is given by the configuration type. For example, when intracochlear electrodes \#2 and \#3 are used as the active and return electrodes, respectively, this is simply referred to as bipolar stimulation at electrode \#2 (BP 2).

Effectiveness of the deafening protocol was assessed by measuring auditory brainstem response (ABR) to acoustic clicks in each ear. Calibrated acoustic assemblies comprising an electrodynamic speaker and a probe-tube microphone were inserted into the cut ends of each ear canal to form a closed system. Condensation clicks $(100 \mu \mathrm{s})$ were delivered via these acoustic systems, and ABR thresholds were measured in both ears. ABR was measured between vertex and ear bar using a small screw inserted into the skull. In all experiments, no ABR response was seen up to the highest intensity tested (110 dB SPL peak).

\section{Stimulus generation and delivery}

All stimuli were generated by a pair of 16-bit digital-toanalog converters $(\mathrm{D} / \mathrm{A})$ at a sampling rate of $100 \mathrm{kHz}$. Stimulus levels were set by custom-built attenuators having a resolution of $0.1 \mathrm{~dB}$. Attenuated outputs of the $\mathrm{D} / \mathrm{A}$ converters were delivered to the intracochlear electrodes via a pair of custom-built, high-bandwidth $(40 \mathrm{kHz})$, optically isolated, constant-current sources. All electric stimuli were made up of $100 \mu$ s biphasic current pulses (cathodic-anodic, $50 \mu \mathrm{s} /$ phase).

\section{EABR measurements}

The EABR was measured by recording the voltage between a screw placed at the vertex of the skull and the ear bars of the stereotaxic device and averaging the responses to 500 stimulus presentations. The stimulus was a biphasic pulse $(50 \mu \mathrm{s} /$ phase, $21 / \mathrm{s}$ repetition rate) with alternating polarity on each stimulus presentation. The alternating polarity allows for the stimulus artifact, which reverses in polarity with the stimulus, to be mostly averaged out from the neural response, which does not reverse polarity with the stimulus. Evoked potentials were amplified $(60 \mathrm{~dB})$ and band-pass-filtered (cutoff frequencies of $100 \mathrm{~Hz}$ and $10 \mathrm{kHz}$ ). The resulting signal was digitized at a sampling rate of $20 \mathrm{kHz}$, and the average waveform (500 responses) was saved to disk. In postprocessing, EABR waveforms were digitally filtered twice (once forward and once reversed) with a 31st-order FIR band-pass filter $(300-3,000 \mathrm{~Hz}$ cutoffs) so that no net delay was added to the signal. The amplitude of Wave 4 was determined for binaural and monaural EABR waveforms by measuring the voltage between the preceding trough and peak of Wave 4 (as indicated by triangles in Fig. 1A-C). Wave-4 amplitude was measured as a function of pulse current for each intracochlear stimulating electrode in bipolar and monopolar configurations and hereafter will be referred to as EABR amplitude. We also calculated the BIC of the EABR by subtracting the sum of the monaural EABR responses from the binaural EABR response (Fig. 1). Note that this definition results in a BIC waveform with the opposite polarity from that used by Melcher (1996) in normal-hearing cats. The BIC consists of a single biphasic wave, and its amplitude was measured between the preceding trough and the peak as shown in Figure 1D.

\section{Inferior colliculus recordings}

Neural activity was recorded simultaneously at multiple locations in the IC using 16-channel silicon probes (NeuroNexus Technologies, 100 or $150 \mu \mathrm{m}$ 

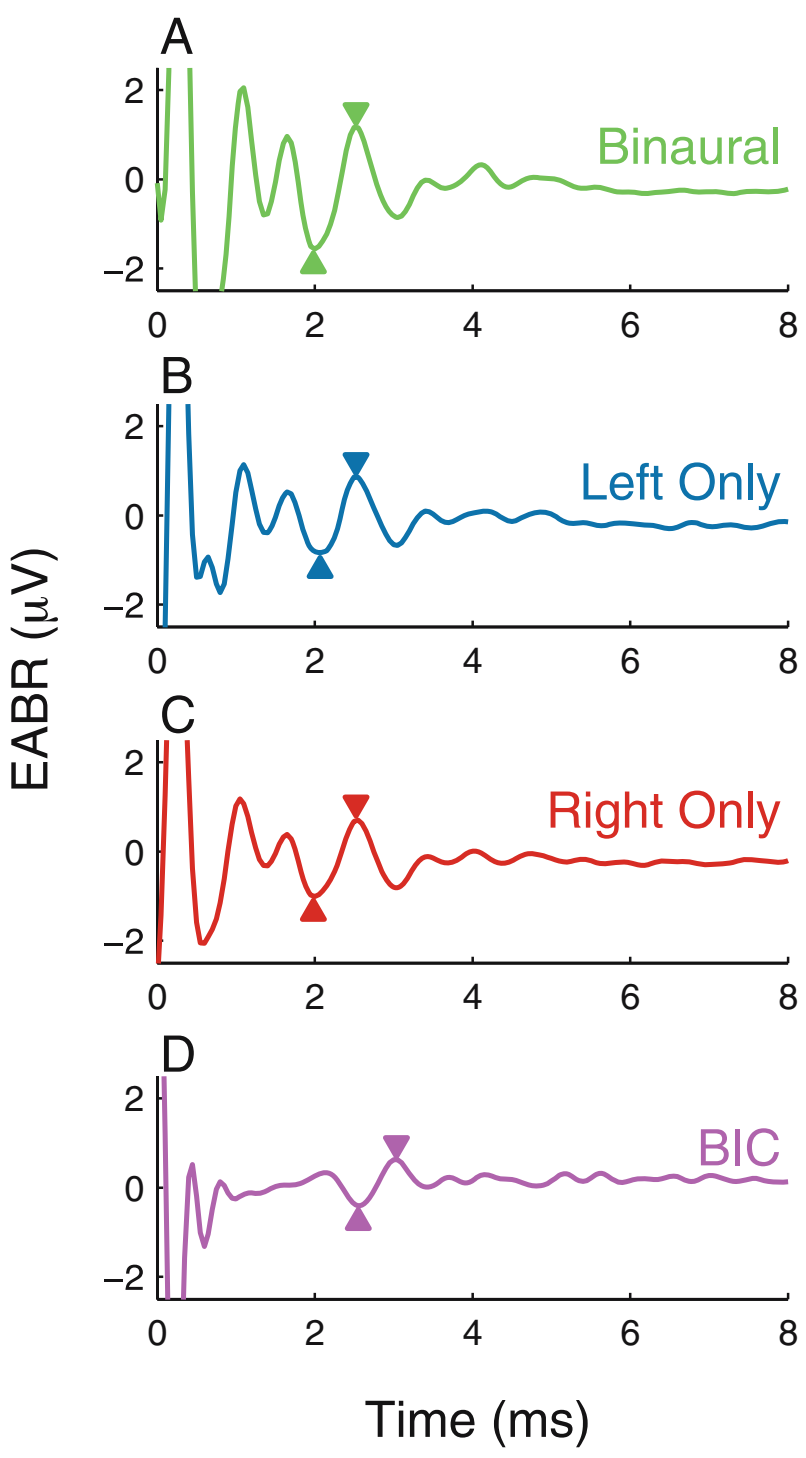

FIG. 1. Measuring the binaural interaction component (BIC). (A-C) EABR waveforms for binaural stimulation and monaural stimulation of each ear with single biphasic current pulses. Waves 2, 3, and 4 are seen, but Wave 1 is obscured because the first $1 \mathrm{~ms}$ of the EABR waveforms contains residual stimulus artifact. Triangles show the times used to measure the peak-peak amplitudes of each response. (D) BIC waveform computed by subtracting the sum of the monaural evoked responses from the binaural response.

linear spacing, $177 \mu^{2}$ site area). The recording probe was advanced along the tonotopic axis of the IC from dorsolateral to ventromedial, which was (the probe) in the coronal plane tilted $45^{\circ}$ off the sagittal plane so as to record activity from neurons covering a highly reproducible wide range of CFs (Merzenich and Reid 1974; Snyder et al. 1990). Neural responses to a single biphasic current pulse $(50 \mu \mathrm{s} /$ phase $)$ were recorded simultaneously from all 16 channels, and the raw waveforms of the signals at each probe site were saved to disk using a sampling rate of $20 \mathrm{kHz}$.
Isolation of neural activity at each site across the 16site recording probe varied depending on the position of the array in the IC. While most sites exhibited fairly uniform local field potentials and multiunit responses, it was not uncommon for two to four sites to show well-isolated single units. To obtain a more uniform neural response quality across sites, responses to 10 stimulus presentations were averaged for each site, thereby deemphasizing the larger voltage excursions reflecting single-unit activity. The average response was then low-pass-filtered at $3 \mathrm{kHz}$, and the RMS amplitude was calculated over a 10-ms window, beginning $2.5 \mathrm{~ms}$ after the stimulus (Fig. 2A). Figure 2A shows filtered average response waveforms for an example recording at one site as a function of stimulus intensity. This neural activity is a combination of multiunit activity $(>300 \mathrm{~Hz})$ and local field potentials $(<300 \mathrm{~Hz})$. The corresponding RMS amplitudes, as calculated from the response waveforms, show monotonic growth with increasing intensity (Fig. 2B).

Neural activation patterns along the tonotopic axis of IC were assessed by plotting neural response for a constant-intensity stimulus as a function of depth of each probe contact along the IC (i.e., probe site). In an effort to improve upon the $100-$ to $150-\mu \mathrm{m}$ resolution of the recorded IC neural activation patterns, the precise location of the response peak was estimated by the following procedure: 1) spatial profiles were smoothed by convolution with a threepoint triangular kernel, 2) the smoothed curves were
A

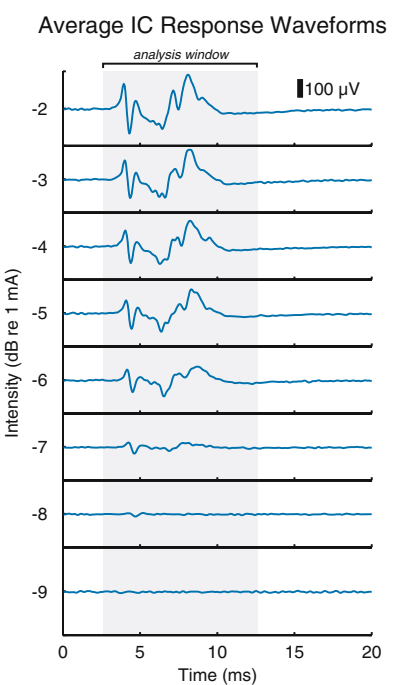

B

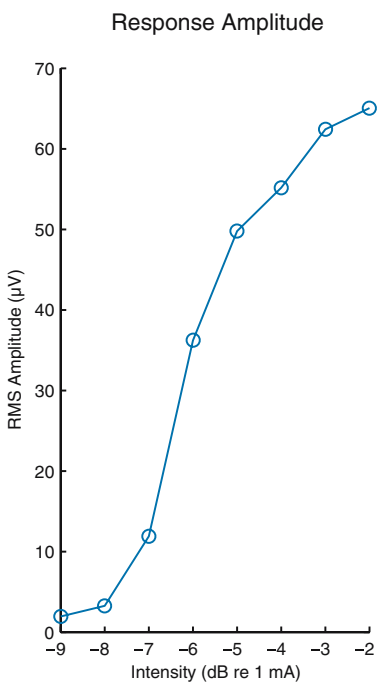

FIG. 2. (A) Average response waveforms recorded in the IC site with a silicon probe for bipolar stimulation of the contralateral ear with a biphasic pulse at eight different levels. Analysis window used to compute RMS amplitude is $10 \mathrm{~ms}$ in duration and starts $2.5 \mathrm{~ms}$ after the stimulus pulse. (B) RMS response amplitude as a function of stimulus level. 
then interpolated with a cubic spline, and 3) the maximum of each interpolated curve was picked as the response peak. The alignment of neural activation patterns elicited by different intracochlear electrodes was compared by measuring the physical distance between estimated response peaks from their respective spatial profile.

\section{RESULTS}

Results are based on EABR recordings in seven deafened cats with bilateral cochlear implants that were used as part of a larger study of single-unit responses in the IC. In these animals, we studied the effect of interaural electrode pairing on the BIC waveform. Parallel recordings at 16-32 sites along the tonotopic axis of IC were made in four of these animals to characterize the spatial patterns of neural activation in the auditory CNS with different intracochlear stimulating electrodes. Best interaural electrode pairings were determined with the evoked responses by finding the interaural electrode pairs that produced the largest BIC amplitude given equivalent strength stimuli. Interaural electrode pairings were also assigned based on the degree of alignment of neural activation patterns in the IC for individual stimulus electrodes. We compare interaural pairings determined with both methods and analyze the correlation between BIC amplitude and neural distance along the tonotopic axis of the IC.

\section{EVOKED POTENTIALS}

Typical monaural EABR, binaural EABR, and BIC waveforms are shown in Figure 1. While increasing stimulus intensity increases the amplitude and decreases the latency of these waveforms, latencies were calculated for stimuli at a standard intensity that evoked a monaural EABR amplitude of 1.5-2.0 $\mu \mathrm{V}$. Mean results are shown in Table 1 for stimulation of 25 interaurally matched electrode pairs in seven animals (see below for method to find interaural electrode matches). The mean latency of the peak of Wave 4 of the EABR was about $2.6 \mathrm{~ms}$ for both binaural and monaural stimulations. The BIC (Fig. 1D) consisted of a single biphasic wave with a trough followed by a peak occurring at latencies of 2.6 and $3.1 \mathrm{~ms}$, respectively. The trough of the BIC is synchronous with Wave 4 of the (monaural or binaural) EABR, whereas the peak of the BIC occurs about $0.5 \mathrm{~ms}$ later. This pattern of relative latencies is similar to that found for acoustic stimulation with
TABLE 1

Latencies of Wave 4 of the EABR and BIC (mean \pm 1 SD)

\begin{tabular}{lc}
\hline \multicolumn{1}{c}{ EABR wave } & Mean latency $(\mathrm{ms})$ \\
\hline Binaural Wave 4 (peak) & $2.58 \pm 0.08$ \\
Monaural Wave 4 (peak) & $2.59 \pm 0.08$ \\
BIC trough & $2.58 \pm 0.14$ \\
BIC peak & $3.11 \pm 0.08$ \\
\hline
\end{tabular}

Data are for 25 matched interaural electrodes in seven animals.

clicks in normal-hearing cats (Caird et al. 1985; Melcher 1996) (Note that Melcher's polarity convention for the BIC is opposite from ours).

Effects of overall stimulus level and interaural level difference on EABR and BIC amplitudes

EABR amplitude always grew monotonically with stimulus level regardless of electrode position in the scala tympani and electrode configuration (e.g., bipolar and monopolar). Mean amplitudes for binaural EABR, monaural EABR, and BIC are shown for bipolar stimulation as a function of monaural stimulus intensity in Figure 3A. Left and right monaural EABR amplitudes are overlapping, whereas binaural EABR is greater and BIC amplitude is smaller than the monaural EABR. The rate of growth of EABR amplitude with level was analyzed for each monaural EABR curve by computing the slope of a straight line fitted to the suprathreshold portion of the curve by the least squares method (mean $R^{2}=0.94, n=105$ ). While thresholds varied between electrodes and animals, with the more apical electrode generally having lower thresholds, the rates of growth of EABR amplitude with level only depended on electrode configuration, not on location of stimulation along the cochlear partition. Rates of EABR growth for monopolar electrode configurations (fitted slope $=0.82 \pm 0.31 \mu \mathrm{V} / \mathrm{dB}$ ) were typically more than twice larger than those for bipolar configurations (fitted slope $=0.21 \pm 0.11 \mu \mathrm{V} / \mathrm{dB}$ ) (Fig. 3B). A two-way analysis of variance (ANOVA) of the effects of electrode number and electrode configuration on the slopes of monaural EABR curves confirms the significant effect of electrode configuration [bipolar vs monopolar, $F(1,102)=30.4, p<0.001]$ and the lack of a significant effect of location of stimulation along the cochlear partition $[F(1,102)=0.08, p=0.77]$. There was no significant interaction between electrode position and electrode configuration $[F(1,102)=$ $3.17, p=0.07]$.

EABR responses were also measured as a function of interaural level difference (ILD). For these measurements, the mean binaural level was held constant 

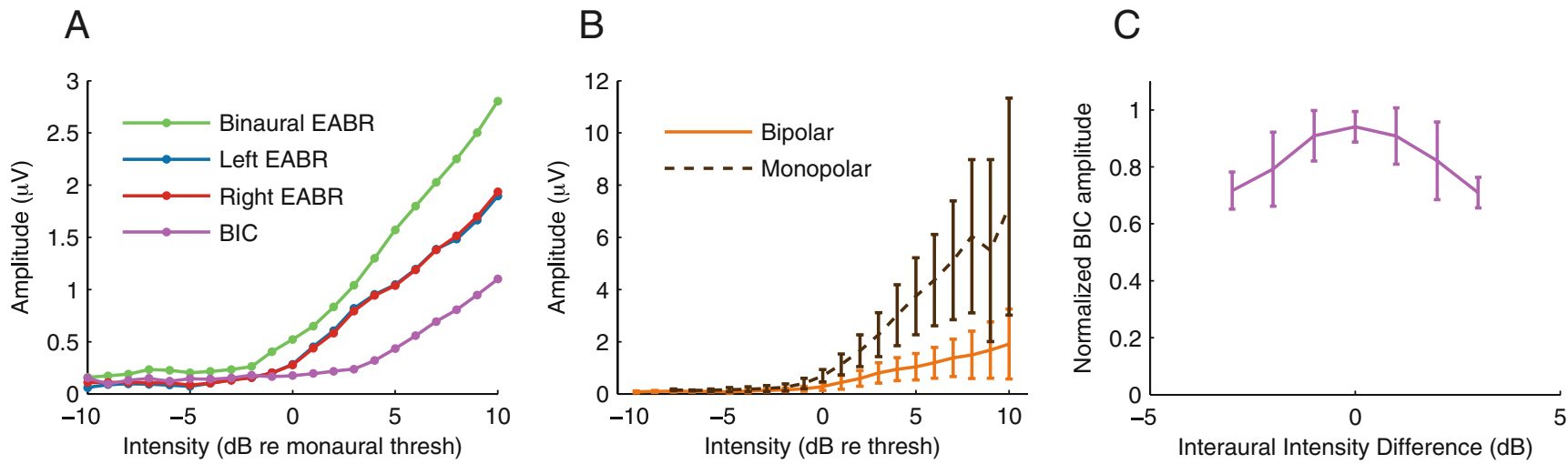

FIG. 3. Effects of stimulus intensity on evoked responses. (A) Mean EABR amplitude-level functions for bipolar stimulation of the cochlea across all animals and electrodes. Increasing stimulus intensity results in greater binaural EABR, monaural EABR, and BIC amplitudes. Intensity is given in decibel re monaural EABR thresholds. (B) Mean monaural EABR amplitude-level functions for bipolar and monopolar stimulations. Error bars show \pm 1 SD. Bipolar stimulation results in a more gradual growth of EABR amplitude than monopolar stimulation. (C) Mean normalized BIC amplitude across all animals as a function of interaural intensity difference (ILD). The 0-dB point for each individual curve is set to where monaural EABR amplitudes are equal. The mean binaural level was kept constant as ILD was varied. BIC amplitude peaks at an ILD of $0 \mathrm{~dB}$.

by changing the level in each ear in equal decibel steps in opposite directions. While monaural EABR amplitudes grow with increasing intensity in each ear, the BIC amplitude is maximal for a given ILD and falls for ILDs in either direction (Fig. 3C). The ILD that produces the maximum BIC amplitude generally corresponded to intensities in each ear that produced similar monaural EABR amplitudes, typically a fixed number of decibels above threshold. Thus, the BIC amplitude peaks when the stimuli in each ear are at the same "effective" intensity. With acoustic stimulation in normal-hearing humans, the BIC amplitude peaks for click stimuli that are centered in the head (Furst et al. 1985; Riedel and Kollmeier 2002). For clicks that are presented simultaneously (zero ITD), this occurs when they are the same level in both ears $(\mathrm{ILD}=0 \mathrm{~dB})$.

Finding the BIC amplitude for binaurally balanced stimuli

Because the BIC amplitude is highly dependent on the intensities in both ears, we needed to find a way to make fair comparisons of BIC amplitude across different interaural electrode pairings. Assuming that monaural stimuli that produce equal amplitude EABRs have the same "effective" stimulus strength, we first found stimulus intensities that elicited the same monaural EABR amplitude and used these intensities in each ear to measure the BIC. The procedure for finding binaurally balanced stimuli is shown in Figure 4A for an example of interaural electrode pair. The intensities for each ear were first picked by measuring monaural EABR amplitude as a function of level and finding the stimulus intensity that evoked an EABR amplitude of 1.5-2.0 $\mu \mathrm{V}$. One ear was designated the "fixed" ear, and its intensity was held constant during subsequent EABR measurements, whereas the intensity in the opposite ear ("varied" ear) was varied over a small range of intensities (usually five $0.5 \mathrm{~dB}$ steps covering a range of $2 \mathrm{~dB}$ ) around the intensity that evoked a similar EABR amplitude as in the fixed ear. For the example in Figure 4A, the right ear intensity is fixed at $5 \mathrm{~dB}$ re $1 \mathrm{~mA}$, and the right EABR amplitude is therefore constant. The EABR amplitude for monaural stimulation of the left ear increases gradually as the left-ear intensity is varied from 7 to $9 \mathrm{~dB}$ re $1 \mathrm{~mA}$. Unlike the case when the mean binaural level is fixed, for which BIC functions peak at a given ILD, here, the BIC increases with increasing level in the varied ear because the mean binaural level is also increasing. The intensity at which the monaural EABR functions cross is assumed to be the intensity for the "varied" ear that matches the effective stimulus strength of the "fixed" ear. Monaural EABR and BIC amplitudes are interpolated by linear regression (solid lines in Fig. 4A), and the BIC amplitude is estimated at the matching intensity of $7.9 \mathrm{~dB}$ (purple arrow).

The above procedure was repeated for five different electrodes in the "varied" ear, whereas the electrode and stimulus level in the "fixed" ear was unchanged to measure BIC amplitude as a function of interaural electrode offset. Figure 4B plots the BIC amplitude for all five pairings between electrode \#3 in the "fixed" ear and each of the five electrodes in the "varied" ear. This curve is called a BIC-electrode function. For the example in Figure $4 \mathrm{~B}$, the BIC amplitude is maximal for electrode \#3 in the "varied" ear which corresponds to an interaural offset of zero 

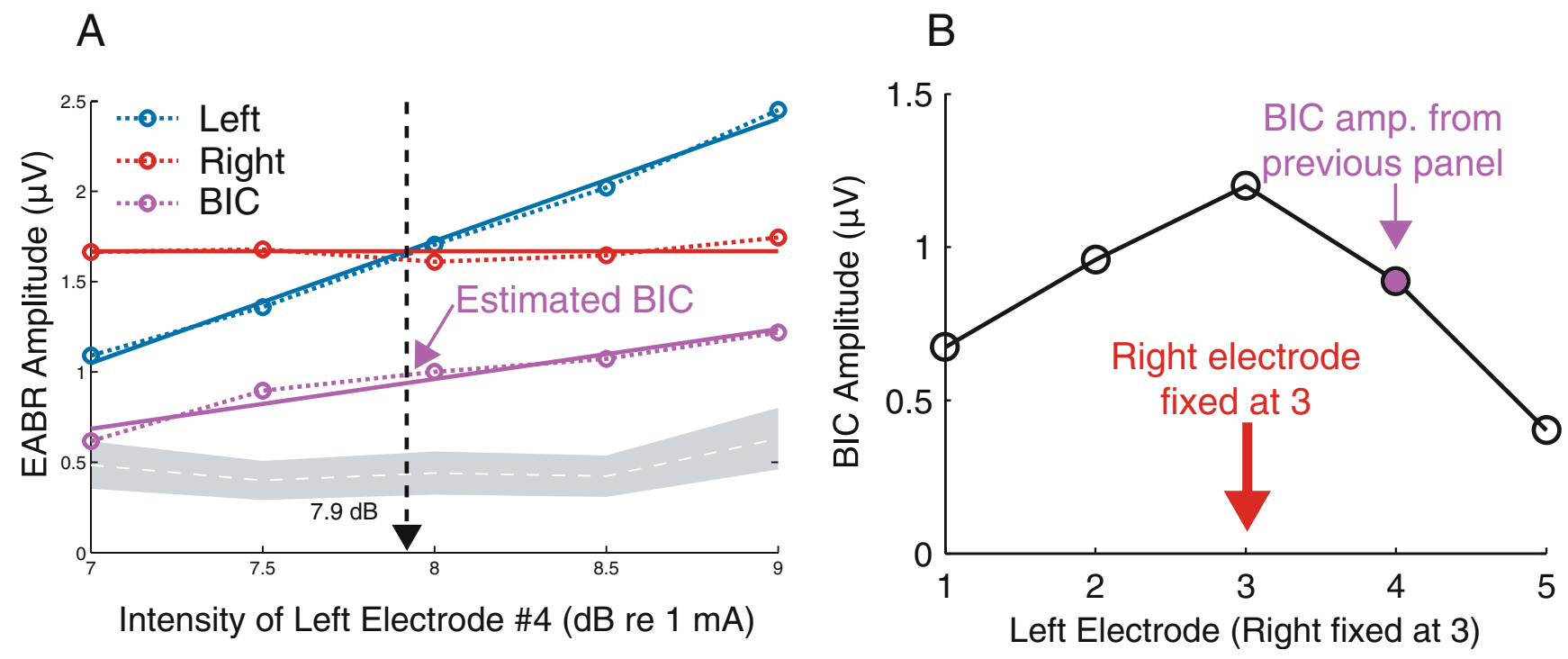

FIG. 4. Estimating the BIC amplitude for matching stimulus strength in interaural electrode pairs. (A) Monaural EABR and BIC amplitudes are measured for a fixed intensity in the right ear $(5 \mathrm{~dB}$ re $1 \mathrm{~mA}$ ) and a range of intensities in the left ear (points fit with linear regression). The leftand right-ear intensities are considered matched when they evoke the same monaural EABR amplitude (7.9 dB in left ear, dashed black line). The BIC amplitude for this interaural electrode pair is measured for the matched intensities (purple arrow). The noise floor of the measurements is indicated by gray shading. (B) BIC amplitude as a function of the active electrode in the left ear, with the right-ear stimulation fixed at BP 3. The BIC amplitude measured in panel $A$ was for the interaural electrode pair right BP 3 and left BP 4 (purple point). The same-level matching procedure was repeated for five different bipolar electrode locations in the left ear.

electrodes. This result is consistent with our goal of achieving the same depths of insertion of the electrode array in the two cochleae.

\section{Dependence of BIC amplitude on interaural electrode offset}

The BIC amplitude was measured for various interaural electrode pairings to test the hypothesis that "matched" interaural electrode pairs produce greater BIC amplitude than "unmatched" pairings. As described in the previous section, BIC amplitude was measured for stimulation at a constant cochlear position and stimulus intensity in one "fixed" ear, whereas the cochlear place of stimulation was systematically varied along the intracochlear array in the other "varied" ear. For each cochlear position, the stimulus intensity in the "varied" ear was adjusted so as to produce balanced stimulation. In total, $25 \mathrm{BIC}-$ electrode functions were measured in seven animals (Table 2). Across the entire set of BIC-electrode curves, the mean monaural EABR amplitude \pm 1 SD was $1.72 \pm 0.23 \mu \mathrm{V}$. The mean difference \pm 1 SD between the intensity in the "fixed" ear and the matching intensity in the "varied" ear was $-1.1 \pm 4.5 \mathrm{~dB}$ re $1 \mathrm{~mA}$. Although in the example of Figure 4 the varied ear was the left one, this was not always the case $(6 / 25$ curves were measured with left ear fixed).

TABLE 2

Breakdown of BIC-electrode data by animal

\begin{tabular}{ccccc}
\hline Animal number & Configurations tested & Number of BIC-electrode curves & $\begin{array}{c}\text { Interaural electrode } \\
\text { offset based on BIC }\end{array}$ & Does BIC predict a consistent offset? \\
\hline 23 & BP, BP+1 & 5 & 0 & Yes (5/5 same) \\
24 & BP & 1 & 0 & N/A (only 1 meas.) \\
25 & BP & 3 & 0 & Yes (3/3 same) \\
28 & BP, MP & 3 & 0 & Yes (3/3 same) \\
30 & BP, MP & 7 & 0 & Almost (6/7 same) \\
32 & BP & 2 & -1 & Yes (2/2 same) \\
34 & BP & 4 & $-1,0,1,1$ & No (3 different offsets) \\
\hline
\end{tabular}

$\mathrm{BP}=$ bipolar, $\mathrm{MP}=$ monopolar. 
The entire set of measured BIC-electrode functions is shown in Figure 5A, with BIC amplitude normalized so that each function peaks at 1 . Each panel shows all the measured BIC-electrode functions for a given fixed active electrode in the array. Data from all animals as well as all electrode configurations are included. Black lines indicate individual BIC-electrode functions, whereas the thick purple line shows the mean curve. In general, the BIC amplitude peaks at a location that is highly dependent on the cochlear location of stimulation in the "fixed" ear. The peak usually occurs for interaural electrode pairings with the same number (zero electrode offsets), which corresponds to electrodes at the same approximate insertion depth. Figure 5B shows all BIC-electrode curves as a function of interaural electrode offset. Most of the curves peak at zero, and the mean curve (thick purple line) is fairly symmetric. On average, an interaural offset of two electrodes $( \pm 1.5 \mathrm{~mm})$ is required to reduce the BIC amplitude by $50 \%$. Figure $5 \mathrm{C}$ shows a histogram of the interaural electrode offsets of the BIC peaks. A large majority of curves $(19 / 25)$ peak at an interaural electrode offset of zero. In no case does the offset exceed \pm 1 interaural electrode distance $( \pm 0.75 \mathrm{~mm})$.

In six of the seven animals, BIC-electrode functions were measured for several electrode locations in the fixed ear (see Table 2). In such cases, one would

A

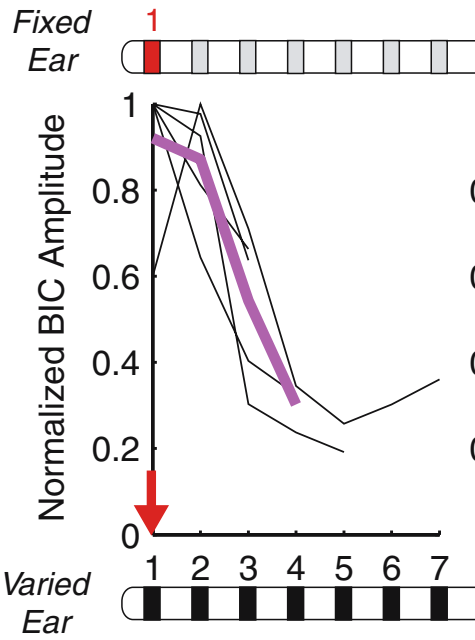

2

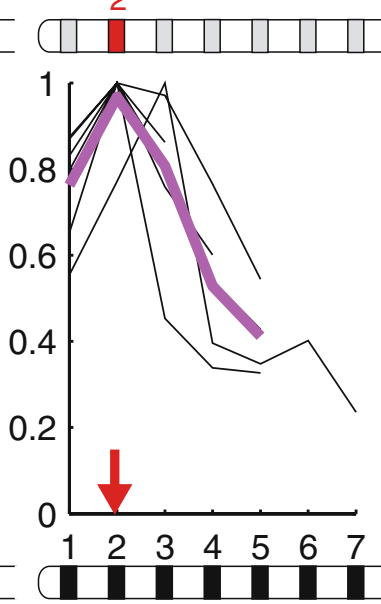

3

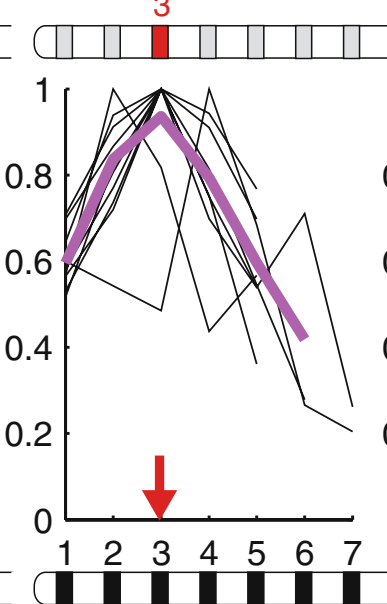

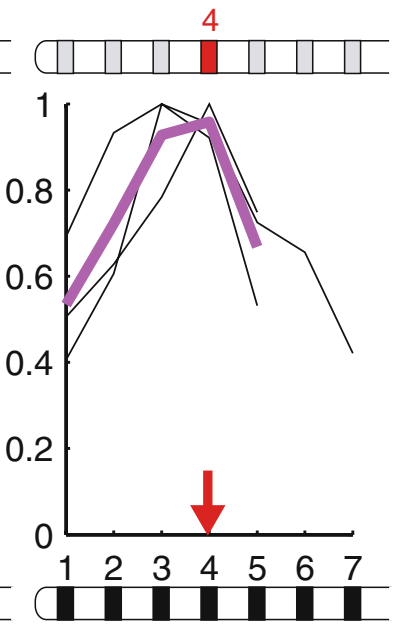

Varied electrode number
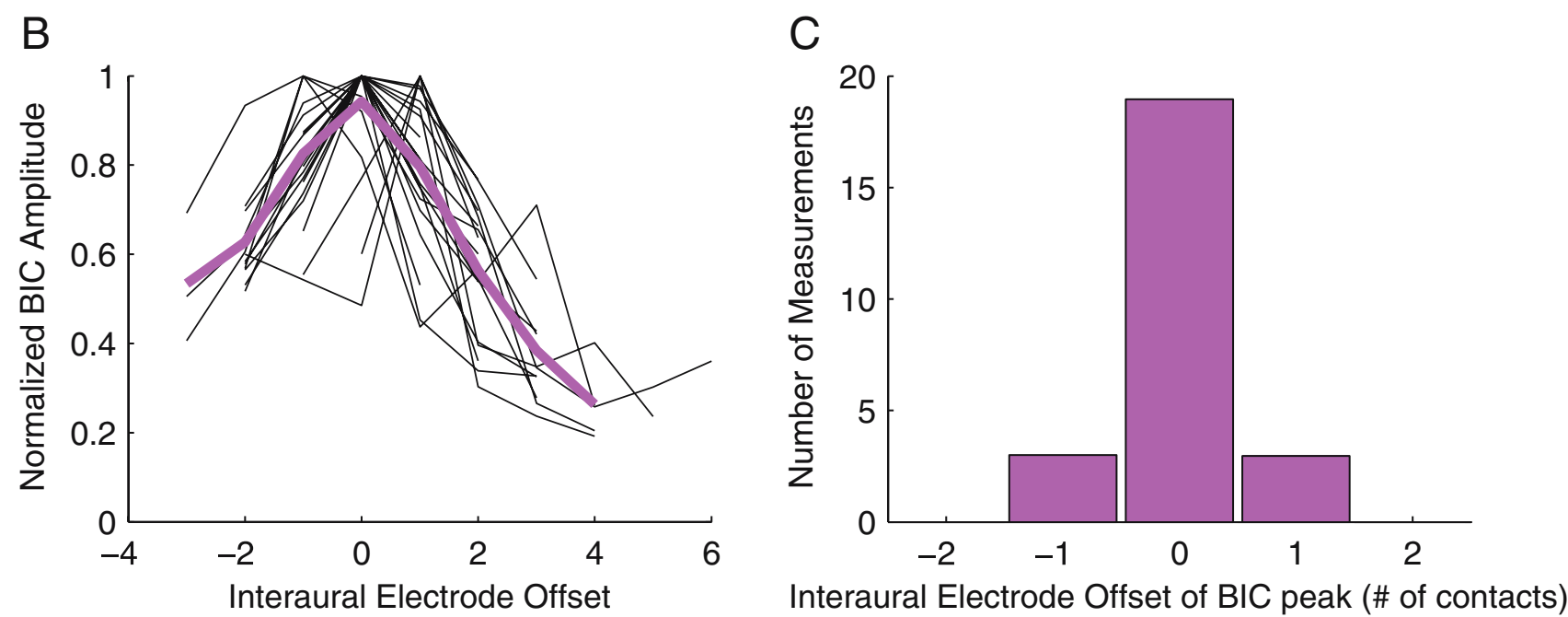

FIG. 5. Effect of interaural electrode offset on BIC amplitude. (A) Complete set of BIC-electrode functions measured in the seven animals (BIC amplitude normalized). Curves are sorted into panels according to the location of the active electrode in the fixed ear (red contact and red arrow). Thin black lines show individual BIC-electrode curves, and thick purple lines show the mean data for each fixed electrode location. All curves are normalized so that the peak BIC amplitude is 1. (B) All BIC-electrode functions superimposed and plotted as a function of interaural electrode offset (varied electrode-fixed electrode). (C) Histogram of the interaural electrode offsets for which BIC peaks in B. 
expect all the interaural electrode offsets that maximize BIC amplitude to be consistent with each other because the spacing of the electrodes in each intracochlear array is constant at $0.75 \mathrm{~mm}$ and the electrode array is relatively rigid. Indeed, all best interaural electrode matches based on the peak of BIC-electrode curves were in agreement with each other for four of six animals, representing 13/24 BICelectrode curves (Animal \#24 is omitted because only one BIC-electrode curve was measured). For the first of the two remaining animals (\#30), six of the seven measurements were consistent with each other. In the last animal (\#34), three of the four measurements indicated inconsistent interaural electrode alignments that differed by as much as two interelectrode distances. The inconsistent interaural electrode offsets in this animal may be due to an unusually low signalto-noise ratio of the BIC measurements or to actual differences in the best offsets. Asymmetries in implantation (as might occur with a bent array in one ear) or cross-turn activation of the auditory nerve are possible reasons for differing offsets at different cochlear locations in the same animal. In three animals, interaural electrode matches were made for different electrode configurations (monopolar, bipolar, etc.) that were tested. For 14 of these 15 cases, interaural electrode offsets were consistent within animals regardless of electrode configuration. Overall, the electrode offsets estimated by maximizing the BIC amplitude are highly consistent in each animal, giving some evidence for the validity of the method. A more direct test of the method for achieving interaural electrode matches is provided in the next section.

\section{INFERIOR COLLICULUS RECORDINGS}

Topographic neural response patterns elicited by the various intracochlear electrodes were measured along the tonotopic axis of the IC in four of the seven animals studied with evoked potentials. This was done to directly test the hypothesis that matched interaural electrode pairs as assessed by the BIC method activate maximally overlapping neural populations. Figure $6 \mathrm{~A}$ shows the orientation of the 16-channel recording probe in the IC, and the presumed tonotopic organization in the central nucleus of the IC with low- to high-CF neurons arranged from dorsal-lateral to ventral-medial (Merzenich and Reid 1974). In one normal-hearing animal, acoustic response maps were measured for pure tones with logarithmically spaced frequencies presented at several intensities. The acoustic response maps were recorded (16 sites at a time) as a function of depth from the surface of the IC, and the CF was estimated at each location. More than 16 IC sites were sampled by moving the recording probe to three different depths. As expected from similar measurements for this trajectory through the IC (Merzenich and Reid 1974; Snyder et al. 1990; Snyder et al. 2004), Figure 6B
A

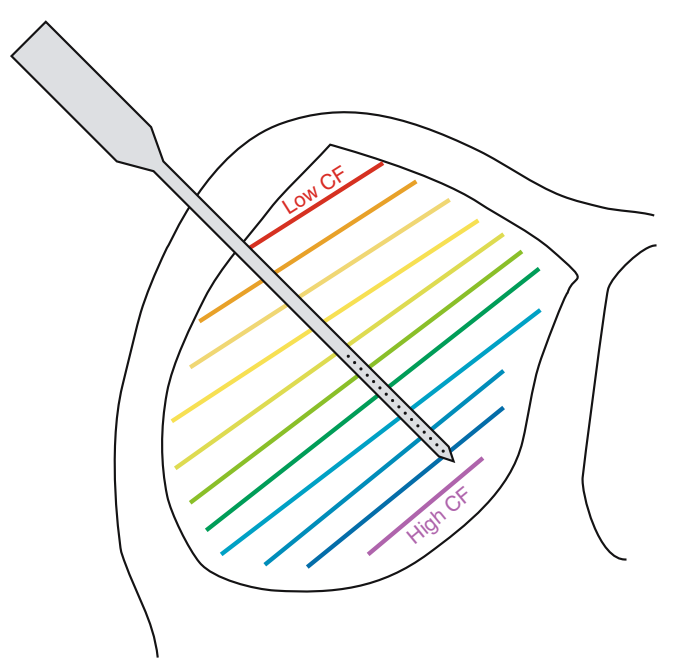

B

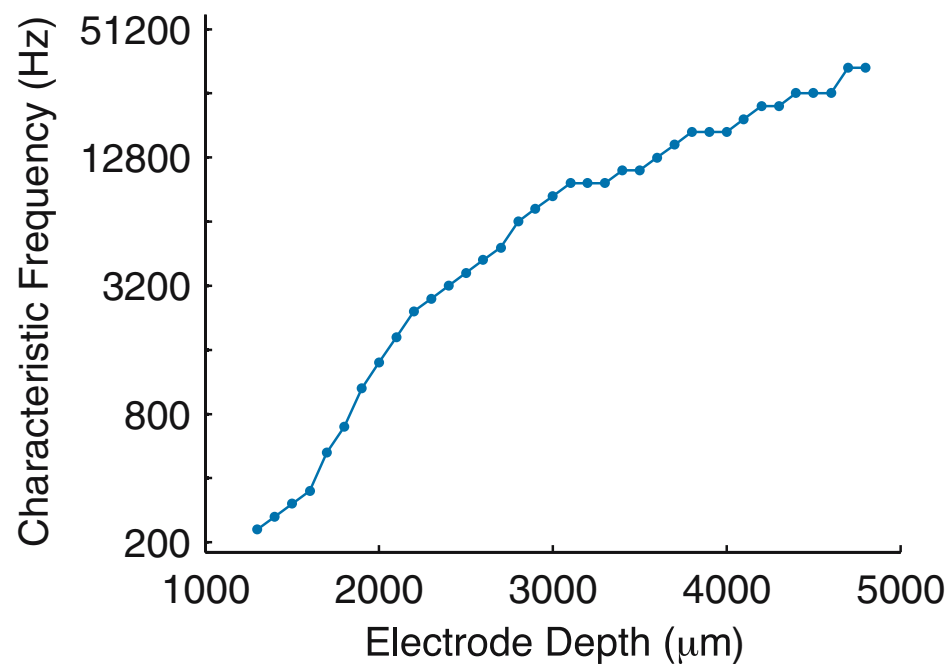

FIG. 6. Acoustic tonotopy of the inferior colliculus. (A) Diagram showing the angle of penetration of the silicon probe into the IC. The probe is in the coronal plane, $45^{\circ}$ from vertical so that the trajectory is from dorsolateral to ventromedial, parallel to the tonotopic arrangement of CFs in the central nucleus of the IC. With this electrode trajectory, low-CF neurons are located at shallow depths from the surface of the IC, and highCF neurons are deeper. (B) CF as a function of electrode depth for one probe penetration in a normal-hearing animal. Data from three different probe positions are combined to cover the entire depth of the IC. 
shows that CF progresses monotonically as a function of recording depth and covers virtually the entire range of hearing over a 4-mm range.

For electric stimulation, the recording probe was fixed at a single position relatively deep in the IC so as to sample higher frequency regions most likely to be stimulated by our electrode arrays, and responses at each of the 16 probe sites were characterized. Example neural spatial response patterns are shown in Figure 7A for bipolar stimulation of the contralateral ear. Each panel shows the spatial response pattern for electric stimulation at a single cochlear

A

\section{Apical}

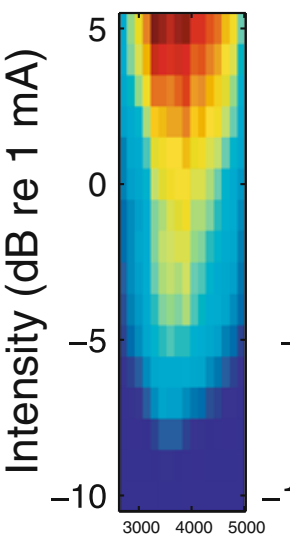

2

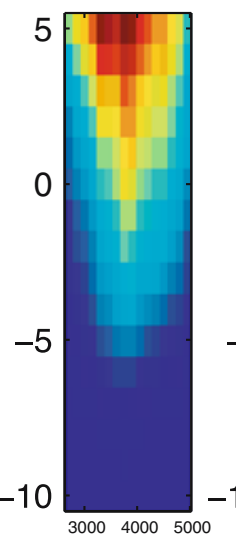

Cl Electrode 1

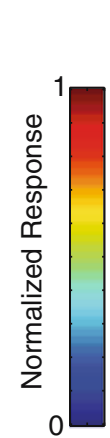

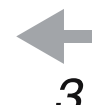
3

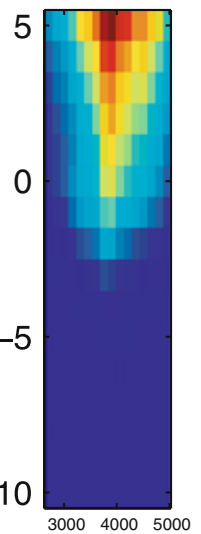

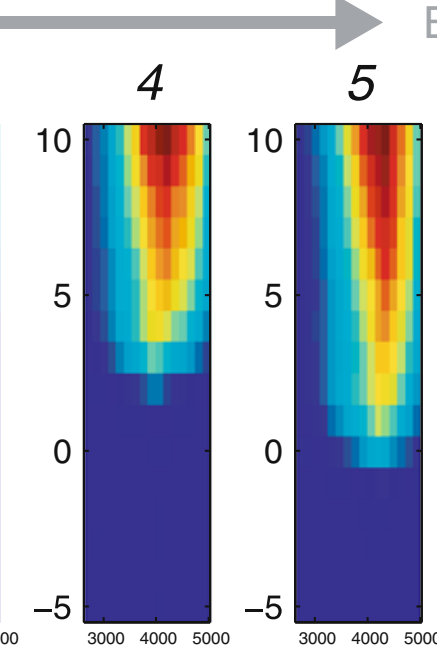

Basal

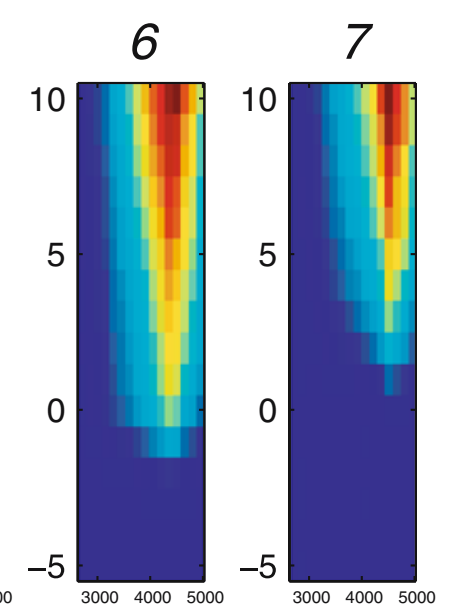

Electrode depth from surface of IC $(\mu \mathrm{m})$

B

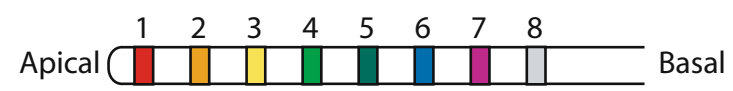

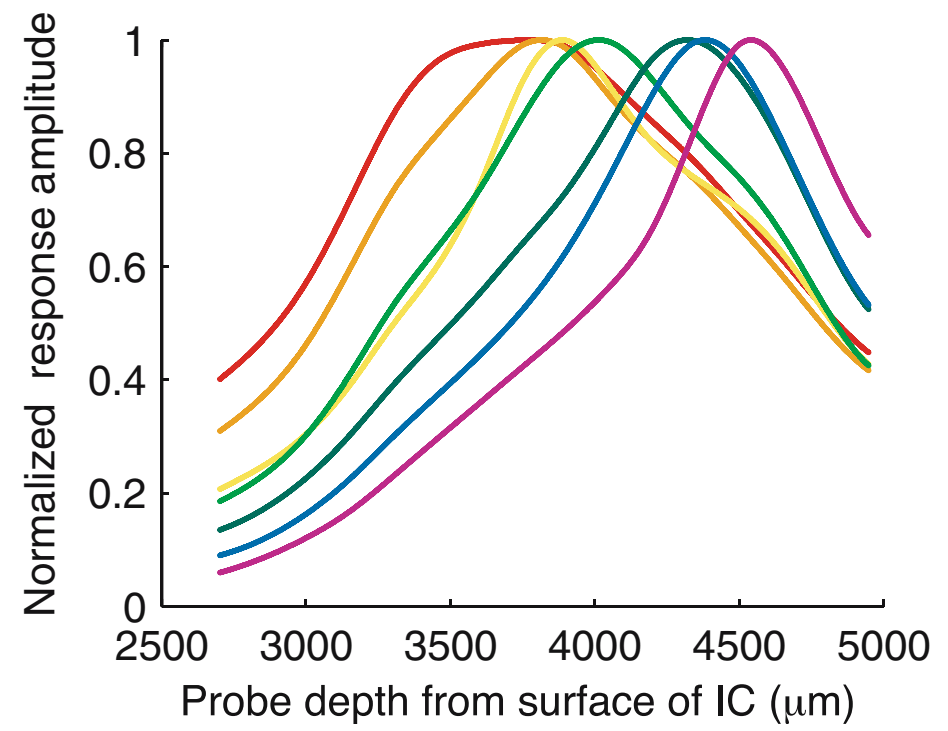

C

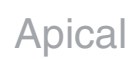

Basal

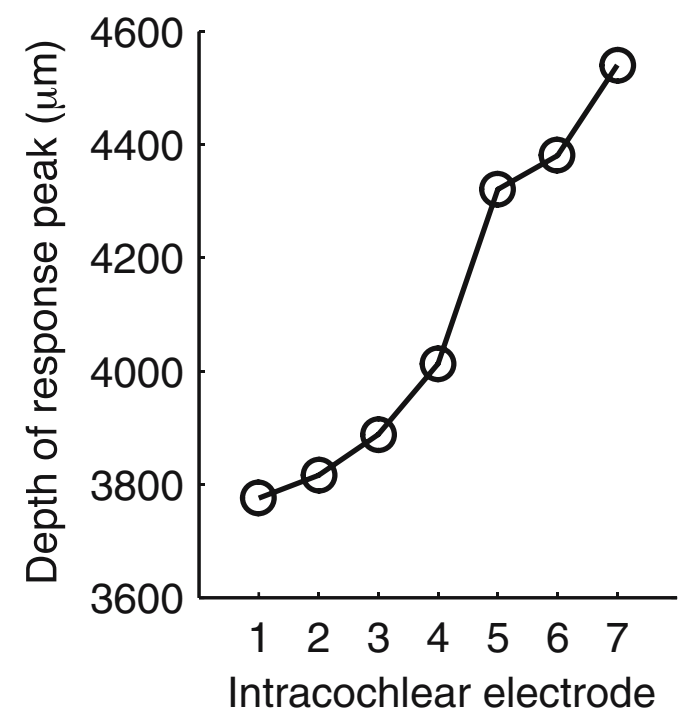

FIG. 7. Neural response patterns with electrical stimulation in the inferior colliculus. (A) Neural response amplitude as a function of position along the IC and stimulus intensity. Color map indicates response amplitude. Each panel shows the neural response pattern for a different bipolar electrode location in the contralateral ear. White horizontal lines indicate stimulus intensities that elicited equal-amplitude EABRs. (B) Neural response amplitude as a function of probe depth in IC for bipolar stimulation with seven electrode pair (see top). These spatial profiles from the neural response patterns shown in $A$ by choosing the stimulus intensity for each electrode to produce the same EABR amplitude. Stimulus intensities are shown in $A$ as white horizontal lines. Curves are smoothed and interpolated with a cubic spline and normalized to their peak. (C) Location of peak of spatial profile in the IC as a function of active intracochlear electrode 
position. From left to right, the panels progress from the most apical cochlear stimulation (BP 1) to the most basal stimulation (BP 7). Within each panel, color indicates the magnitude of the neural response as a function of IC depth (horizontal axis) and stimulus intensity (vertical axis). As stimulus intensity increases, the width of the response pattern increases, indicating a broadening of the neural population excited by the more intense stimuli. Moreover, basalward shifts in the cochlear position of stimulation produce neural response patterns with loci at deeper locations in the IC, consistent with the tonotopic map.

The location of the peak of the neural response patterns was used to quantify the relative position of the response along the tonotopic axis of IC. For each stimulus configuration, the peak of the neural response pattern was estimated at the same stimulus intensity used for the BIC measurements. Figure 7B shows the smoothed spatial profiles at fixed intensities derived from the spatial response patterns in Figure 7A. The stimulus intensities are the same as used for the BIC-electrode measurements and are indicated by horizontal white lines in Figure 7A. While the widths and shapes of each spatial profile in Figure 7B are different, the locations of the activation peaks from this example show an orderly progression to increased depths in the IC for more basal stimulation of the cochlea (Fig. 7C).

\section{Interaural alignment of IC activation patterns}

The location of the peak response of IC activation patterns was used to compare neural activation patterns produced by different interaural electrode pairs. The distance between the response peaks from each electrode in an interaural pair measures the degree of alignment between their respective neural activation patterns and is referred to as the "neural distance". Figure 8A illustrates how the neural distance is measured between the neural activation patterns produced by stimulation at one cochlear location (BP 2) in the "fixed" ipsilateral ear and at several cochlear locations in the "varied" contralateral ear. This figure also shows that the widths of the spatial patterns of IC activation are similar for ipsilateral and contralateral stimulations. The neural distance is plotted as a function of stimulus electrode in the "varied" ear in Figure 8B. The electrode in the "fixed" ear (BP 2) is indicated by the arrow. The best interaural electrode match corresponds to the minimum of the neural distance. For this particular example, the neural distance is minimal when the "varied" electrode is BP 2, corresponding to a zero interaural electrode offset.

When interaural electrode matches were made for several fixed electrodes in the same animal, the question arises as to whether these matches are consistent with each other, as would be expected for rigid electrode arrays. A total of 16 neural distance curves were measured in four animals. In 3/4 animals, best electrode matches based on the minimum of the neural distance functions had mostly consistent offsets (10/12 consistent measurements). The remaining animal (\#34) had inconsistent offsets based on neural distance (three different offsets with four measurements, differing by as much as two interelectrode

\section{A}

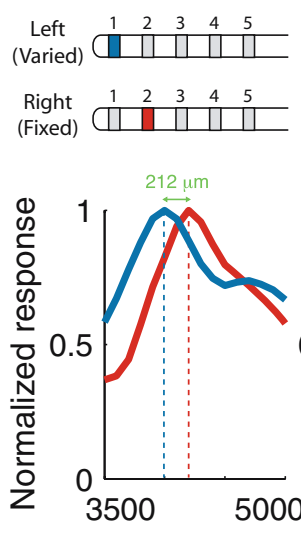

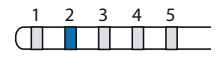
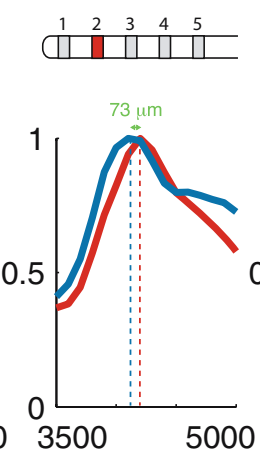

Probe depth from surface of IC $(\mu \mathrm{m})$

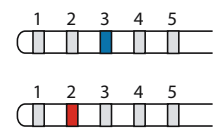

B
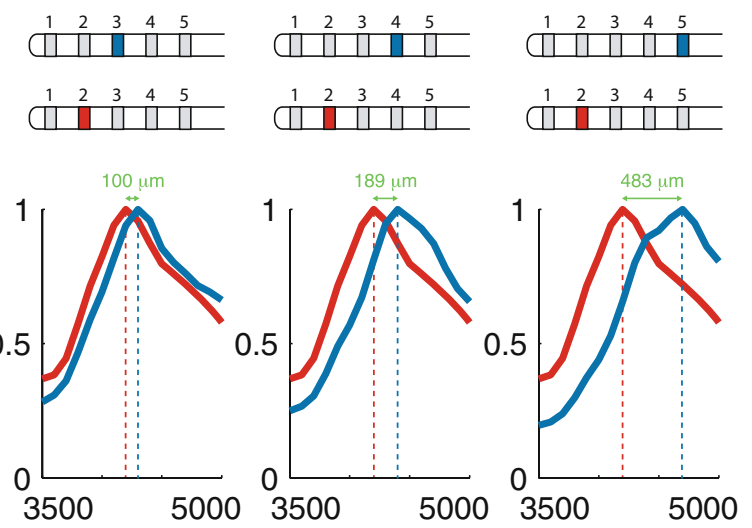

000

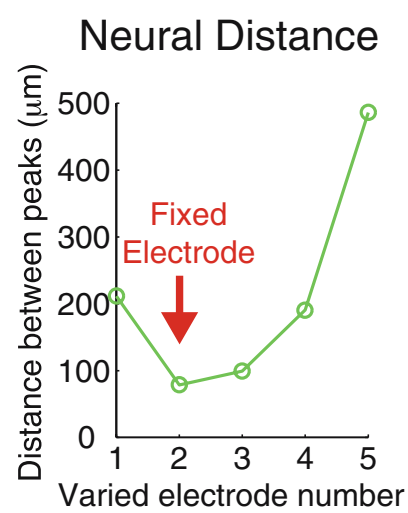

FIG. 8. Matching interaural electrode pairs from IC neural responses patterns. (A) Each panel shows the spatial response profile in the right IC for monaural stimulation of each ear (bipolar configuration). Stimulation in the right/ipsilateral cochlea is fixed at BP 2 , whereas stimulation in the left/contralateral ear differs for each panel. The neural distance is calculated between the peaks of the right and left responses for each interaural pairing. (B) Distance between response peaks as a function of the varied electrode in the left/contralateral ear (location of fixed electrode in right ear indicated by red arrow). The neural distance is minimal at BP 2 in the varied ear. 


\section{Example Comparison}

\section{A}
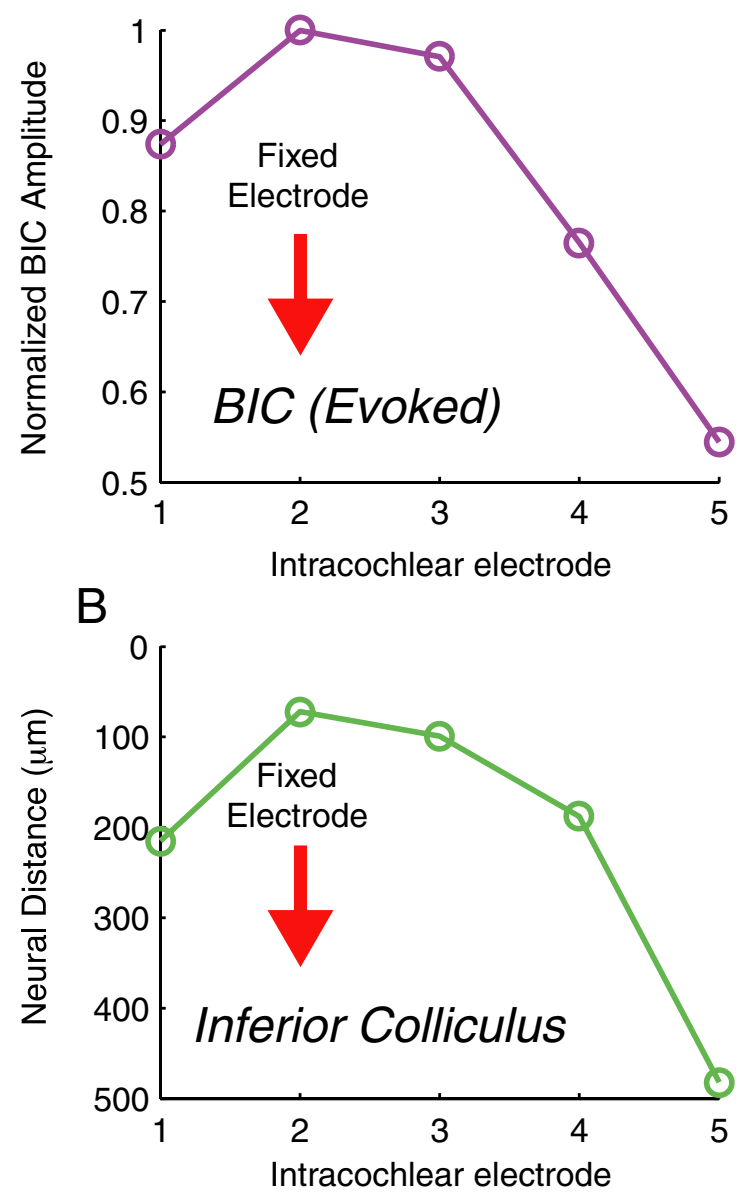

Population Comparison (4 cats; 16 fixed electrodes)
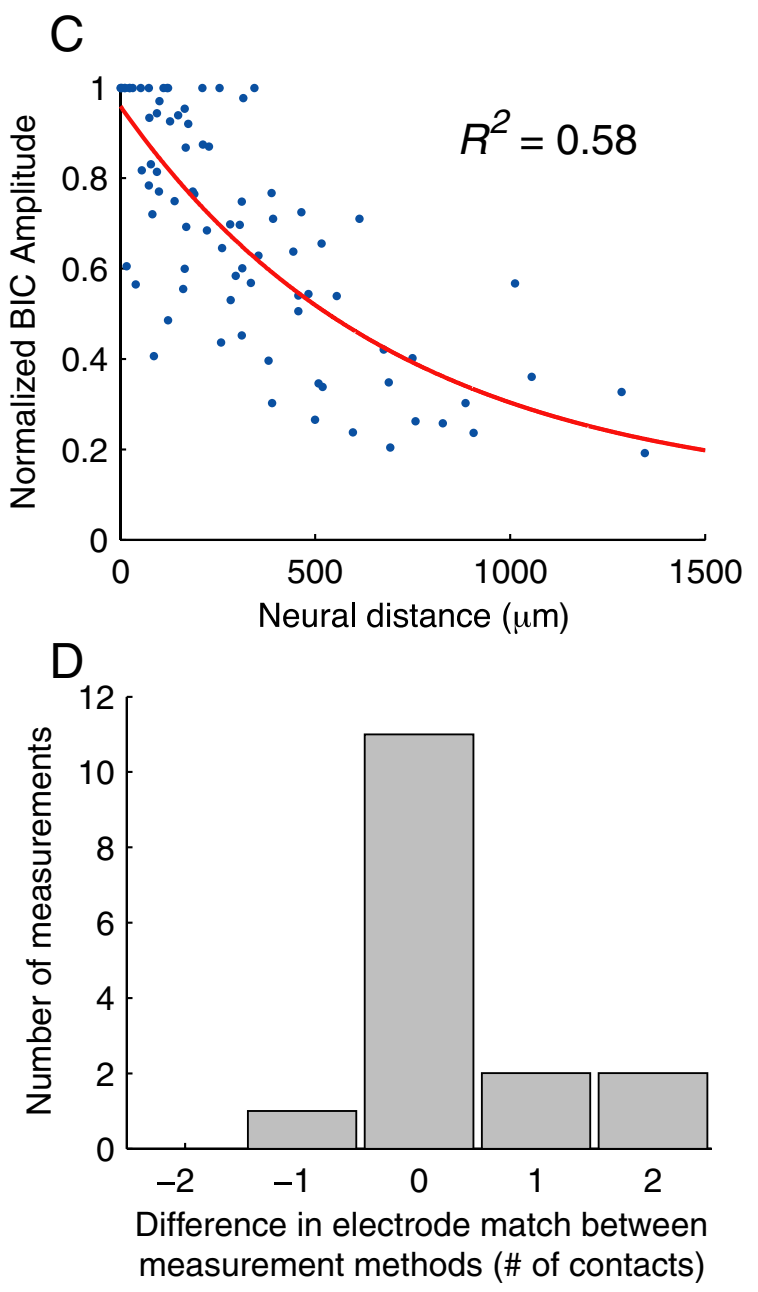

FIG. 9. Comparison of interaural electrode matches based on evoked potentials and IC neural recordings. (A) BIC-electrode curve for fixed electrode BP 2 (indicated by arrow) in one animal. (B) Flipped neural distance function for same animal and electrodes as in panel $A$ (flipped version of Fig. 8B). (C) Normalized BIC amplitude versus neural distance for all data points measured in the same animals and same configurations. Data from four animals and 16 fixed electrodes are included. Solid curve shows best-fitting decaying exponential $\left(R^{2}=0.58\right.$, space constant $=700 \mu \mathrm{m}$ ). (D) Histogram of differences in interaural electrode matches between the two measurement methods. The 11/16 matches are in agreement between the two methods.

distances). This is the same animal that has inconsistent interaural electrode matches based on the BIC.

Comparison of interaural alignments based on EABR and IC activation patterns

The distance between neural activation peaks was measured for all of the interaural electrode pairings tested with evoked potentials for four of the seven animals in which BIC-electrode curves were measured. Figure 9A shows the BIC-electrode function for the same animal and configuration as for the IC data shown in Figure 8. The neural distance function from Figure $8 \mathrm{~B}$ is replotted in Figure $9 \mathrm{~B}$ with the vertical axis inverted. The shapes of the BIC-elec- trode curve and the corresponding neural distance function are strikingly similar. The maximum BIC amplitude and the minimum neural distance both occur at the same place, and thus an interaural electrode match based on either measurement would be the same (i.e., electrode \#2 in the "varied" ear matches electrode \#2 in the fixed ear).

The relationship between interaural electrode alignments based on evoked potentials and neural activation patterns in the IC is examined by plotting normalized BIC amplitude against neural distance measured with the same stimulus configurations in the same animals (Fig. 9C). Note that Figure 9A, B contributes five points to Figure 9C. Across the entire set of data, normalized BIC amplitude and neural 
distance are negatively correlated, with normalized BIC amplitude tending to be large when neural distance is small. Nearly $60 \%$ of the variance $\left(R^{2}=0.58\right)$ in BIC amplitude can be accounted for by a decaying exponential fit to the data $(\mathrm{BIC}=$ $0.86 e^{-\mathrm{nd} / 700}+0.09$, where nd is the neural distance in micrometers). An exponential curve was chosen because it approximates the predicted dependence of the electric potential with distance along the cochlea for simple transmission line models of the cochlea (Black and Clark 1980).

Figure 9D shows a histogram of the differences between interaural electrode matches based on maximum BIC amplitude and those based on minimum neural distance in IC. The matched interaural pairs are in agreement for the majority of cases (11/ 16 fixed electrodes in four animals). The difference between interaural electrode matches with the two methods never exceeds two electrodes $(1.5 \mathrm{~mm}$ in the cochlea). Most of the inconsistencies between the two methods ( $3 / 5$ of interaural pairs not in agreement) came from the same animal (\#34) that had inconsistent interaural electrode offsets with both the $\mathrm{BIC}$ and the neural distance metrics. Both cases where the disagreement reached two electrodes also came from this same animal.

\section{COMPARISON OF BIPOLAR AND MONOPOLAR STIMULATIONS}

So far, our analyses have grouped all the data together regardless of electrode configuration. This was true for both the dependence of BIC amplitude on interaural electrode separation and the spatial patterns of neural activation in IC. A large number of studies of auditory neurons have provided evidence that bipolar stimulation produces more restricted patterns of activity along the tonotopic axis than monopolar stimulation (Merzenich and White 1977; van den Honert and Stypulkowski 1987; Snyder et al. 1990; Kral et al. 1998; Bierer and Middlebrooks 2002; Snyder et al. 2004). Here, we compare the spatial selectivity of both BIC-electrode functions and IC activation patterns for bipolar and monopolar electrode configurations. Our results suggest a slightly different interpretation of the differences between bipolar and monopolar stimulus than previous studies.

\section{Dependence of BIC amplitude on interaural electrode offset}

The second column of Table 2 lists the electrode configurations tested in each animal. Selectivity of BIC-electrode functions was analyzed by plotting normalized BIC amplitude as a function of the absolute value of the cochlear distance from the peak in the BIC-amplitude curves (i.e., BIC-electrode curves are shifted so that they peak at an offset of zero as in Fig. 5B). Figure 10A, B shows the decrease in BIC amplitude with increased interaural electrode offset for bipolar and monopolar stimulations, respectively. On average, BIC amplitude falls to $50 \%$ of its peak value at an offset of about two electrodes $(\sim 1.5 \mathrm{~mm}$ cochlear distance $)$ for both bipolar and monopolar stimulations. Figure 10C shows that, contrary to expectations, the mean BIC amplitudes depend similarly on interaural electrode offset for both electrode configurations. A two-way ANOVA with electrode configuration and interaural

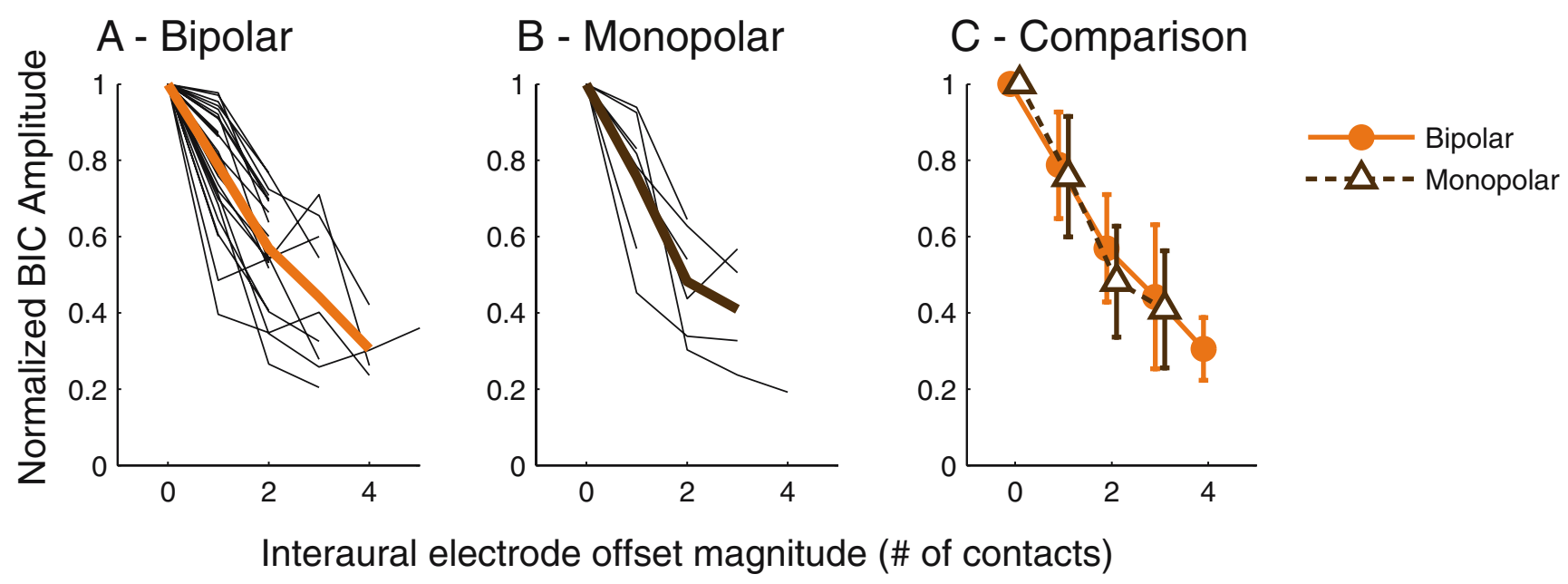

FIG. 10. Comparison of BIC-amplitude falloff with electrode offset for bipolar and monopolar stimulations. (A-B) Thin black lines are individual BIC-offset curves, and thick colored lines are mean values. Curves are shifted so that BIC peak is at zero. (A) Bipolar stimulation. (B) Monopolar stimulation. (C) Comparison of mean bipolar (solid line with closed circles) and monopolar (dashed line with open triangles) stimulations. Error bars indicate $\pm 1 \mathrm{SD}$. 
cochlear offset as factors confirms that, while electrode offset has a significant effect on normalized BIC amplitude $[F(1,107)=193, p<0.001]$, there is no significant effect of electrode configurations $[F(1,107)=0.00, p=0.99]$ and no significant interaction between offset and configuration $[F(1,107)=0.02$, $p=0.28]$. This result suggests that, at the stimulus levels tested, bipolar and monopolar stimulations produce similarly selective neural activity in binaurally sensitive brainstem auditory neurons.

\section{Spatial extent of neural activation patterns in IC}

To more directly test the hypothesis that the spatial widths of neural excitation produced by bipolar and monopolar stimulations may be comparable, neural response patterns recorded in the IC of three animals were analyzed separately for the two configurations. Responses to monaural electric stimulation were recorded at multiple sites along the tonotopic axis of the IC for several stimulus intensities as previously shown in Figure 7A for bipolar stimulation. For each stimulus intensity and stimulating electrode configuration, we determined both the peak response amplitude over all IC recording sites and the spatial extent of the response at half the peak amplitude (the "halfwidth"). Figure 11A shows the mean peak response amplitude as a function of intensity separately for bipolar and monopolar configurations. The mean is taken over all animals and locations of the active electrode. The monopolar response grows faster than the bipolar response by about a factor of 2 , consistent with the growth of EABR in Figure 3B. However, mean halfwidths (Fig. 11B) are comparable for the two electrode configurations for intensities up to $5 \mathrm{~dB}$ above threshold. For higher intensities, halfwidth is greater for monopolar stimulation than bipolar stimulation. A two-way ANOVA on halfwidth, with intensity and electrode configuration as factors, reveals a significant effect of intensity $[F(1,236)=67.3$, $p<0.001]$, no significant effect of electrode configuration $[F(1,236)=1.67, p=0.19]$, and a significant interaction between intensity and electrode configuration $[F(1,236)=8.24, p<0.01]$. This interaction suggests that electrode configuration has an effect on halfwidth only for certain intensities. Restricting the two-way ANOVA to intensities between 0 and $5 \mathrm{~dB}$ re threshold shows only a significant influence of intensity $[F(1,184)=21.6, p<0.001]$ on halfwidth, with no significant effect of electrode configuration $[F(1,184)=0.01, p=0.98]$ nor interaction between configuration and intensity $[F(1,184)=0.01, p=0.94]$.

In an effort to compensate for the difference in growth of responses as a function of intensity for the two configurations, halfwidth is replotted as a function of the peak response amplitude in Figure 11C. Over the range of response amplitudes that overlap between bipolar and monopolar stimulations, the widths of the responses are comparable. These results indicate that, while neural response patterns are wider with monopolar stimulation than with bipolar stimulation for intensities well above threshold, widths are comparable at lower intensities and are similar at intensities that elicit the same response amplitude.

\section{DISCUSSION}

This study investigated a novel method, first suggested by Pelizzone et al. (1990), which uses evoked potentials for finding the relative positions of bilaterally implanted intracochlear electrodes and assign-
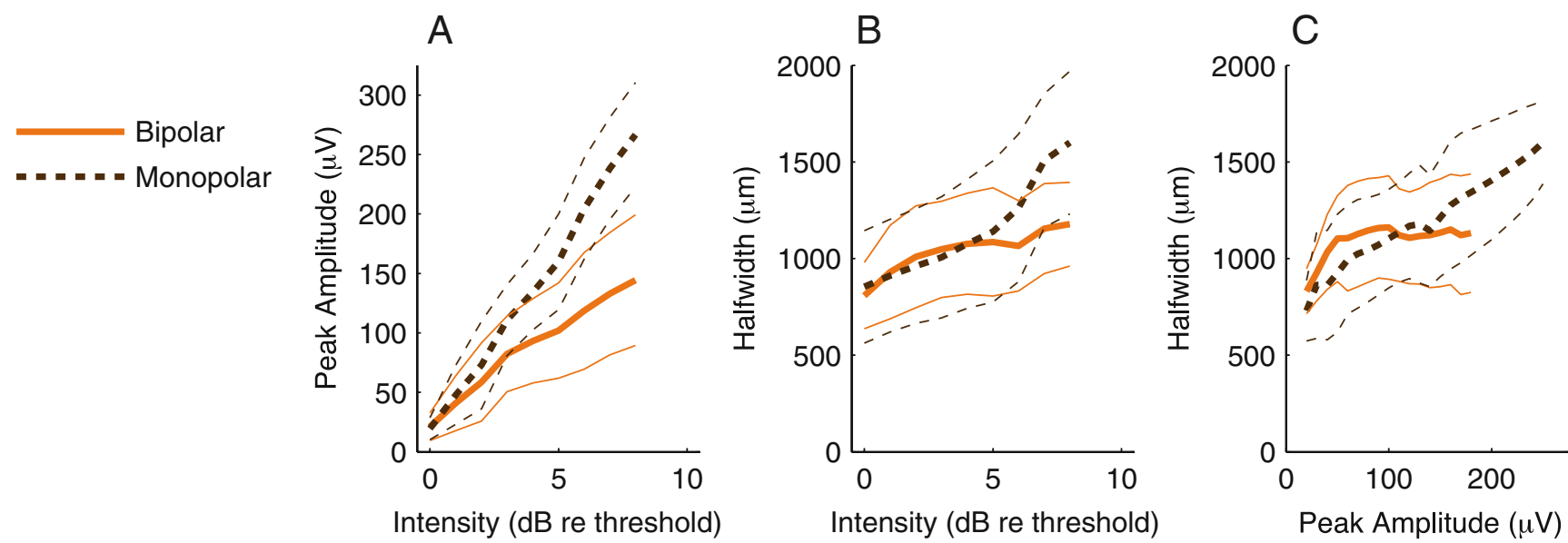

FIG. 11. Comparison of neural response patterns for bipolar and monopolar electrode configurations. Thick lines show means, and thin lines indicate \pm 1 SD from the mean. (A) Peak amplitude of neural activation patterns versus stimulus intensity for bipolar (solid lines) and monopolar (dashed lines) stimulations. The mean is taken over all animals and stimulating electrodes. (B) Halfwidth of neural activation patterns versus stimulus intensity for both configurations. (C) Halfwidth versus peak amplitude of neural activation patterns. 
ing interaural electrode matches. The BIC of the EABR was found to be maximal for stimuli presented at interaural pairs of electrodes in the same intracochlear position when stimulus strength was balanced. Neural response maps in the IC support the hypothesis that BIC-matched interaural electrode pairs stimulate maximally aligned populations of neurons in the auditory CNS.

The presence and characteristics of the BIC response with bilateral electric stimulation of the cochlea are significant findings in this study. Similar to acoustic stimulation in normal-hearing cats (Caird et al. 1985; Melcher 1996), the EABR Wave 4 and the trough of the BIC waveform are synchronous with bilateral electric stimulation of interaurally matched electrodes (Table 1). As with acoustic stimulation in cats (Sontheimer et al. 1985) and humans (Furst et al. 1985; Riedel and Kollmeier 2002), the BIC amplitude decreases by introducing an ILD with electric stimulation (Fig. 3C). The BIC amplitude also decreases with increasing interaural electrode separation (Fig. 5), suggesting that the BIC reflects binaural interactions occurring between specific tonotopic channels. This orderly behavior of the BIC suggests that the binaural circuits of the brainstem work normally in response to electric stimulation in an acutely deafened animal preparation.

\section{Variability of thresholds and using EABR amplitude to match stimulus strength}

An important aspect of measuring meaningful BICelectrode curves in this study was adjusting stimulus levels at each intracochlear electrode so that the stimuli have similar effective strengths at both ears. This is not trivial with cochlear implants because thresholds can vary substantially between electrodes and ears by as much as $20 \mathrm{~dB}$ (Pfingst and Xu 2004). In acutely deafened animals such as ours, variability of threshold is presumably due primarily to the different positioning of the stimulating electrodes relative to the excitable neural elements. In our experiments, the intracochlear electrode array was often repositioned if initial tests of threshold were deemed too high. This was done by pushing the array closer to the modiolus with cotton that filled the space toward the lateral wall of the scala tympani and resulted in lowered thresholds. We assumed that stimuli presented at different cochlear positions have the same effective stimulus strength when they evoke the same Wave- 4 amplitude in the EABR. While it is not known whether this method results in perceptually loudness-balanced stimuli, it was effective for getting consistent results in the BIC-electrode measurements.

While our BIC-based method for making interaural electrode matches appears to work well, the careful level balancing it requires may make it too time-consuming for clinical use. If such a method was adopted in humans who cannot make accurate loudness judgments, such as infants, there may not be enough time to measure the BIC for multiple intensities in the "varied" ear in an attempt to balance stimulus level across the two ears. To address this issue, we repeated the BIC-electrode curve analysis using only the data from a single intensity for each electrode in the "varied" ear. This intensity was chosen to evoke an EABR amplitude of approximately 1.5-2.0 $\mu \mathrm{V}$, as done for the "fixed" ear, based on measurements of monaural EABR amplitude as a function of level using relatively coarse steps $(2 \mathrm{~dB})$. We found that the interaural electrode offsets obtained by the abbreviated method matched those obtained by the full method for 20/25 BIC-electrode curves measured. The remaining $5 / 25$ offsets differed by at most a single electrode from those predicted with the full procedure. Thus, the abbreviated method for making interaural electrode matches based on the BIC would take significantly less time than the full method at the cost of an increased error rate of about $20 \%$. The abbreviated method still requires a relatively time-consuming measurement of monaural EABR as a function of level to choose an appropriate intensity for each electrode. Because determination of electrode threshold is already a requirement for programming the speech processor, the method might be further expedited by measuring the BIC at a fixed level above threshold for each electrode in an interaural pairing. For patients where behavioral thresholds are unavailable, threshold may be based on electrically evoked compound action potential or EABR measurements (Gordon et al. 2004).

\section{Topographic neural response maps in the IC}

Several studies have measured neural activity at multiple sites along the tonotopic axis of IC and auditory cortex in response to monaural stimulation through a cochlear implant (Snyder et al. 1990; Bierer and Middlebrooks 2002; Middlebrooks and Bierer 2002; Snyder et al. 2004). In these studies, the spike rate of multiunit activity was used to define spatial activation patterns with various intracochlear electrode positions and configurations. Here, we used RMS amplitude of the averaged response to assess the combined response strength of both local field potentials and synchronous multiunit activity at each recording site. Using RMS amplitude resulted in more consistent measurements across recording sites that were less influenced by well-isolated single units present at a minority of the probe sites. This analysis was also chosen because the RMS metric closely 
parallels the continuous increase in response amplitude with increasing stimulus intensity seen in the EABR, which is generated from the synchronous activity of many neurons (Fig. 2). Because the IC has a prominent tonotopic organization, this metric was appropriate for characterizing the place and spread of neural activity for different intracochlear electrodes and configurations. However, recordings of multiunits and local field potentials did not show any significant modulation with the ITD of pulse stimuli, whereas ITD sensitivity was seen in the majority of well-isolated IC single units in the same animals (unpublished observations). Thus, the RMS metric was good for the purpose of comparing spatial activity patterns in the IC but not for characterizing ITD sensitivity of IC neurons.

One limitation of our approach is that the spatial resolution of the recording probe along the tonotopic axis of the IC was restricted to $100-150 \mu \mathrm{m}$. In practice, the location of the peak response could be estimated with greater spatial precision by cubic spline interpolation of the spatial response profile. A simplification made in the comparison of neural activation patterns across ears is that only the relative locations of the response peaks were taken into account and not the shapes of the response patterns. Consideration of the shape of the response patterns may be important for irregularly shaped or asymmetric response patterns sometimes observed in our data.

\section{Comparisons of bipolar and monopolar stimulations}

An unexpected result was the comparable spatial selectivity of BIC-electrode curves with bipolar and monopolar stimulations for stimulus levels within $5 \mathrm{~dB}$ of threshold. Neurophysiological studies with cochlear implants have almost universally reported that monopolar stimulation is relatively broad and provides less spatial selectivity in its activation patterns compared to bipolar stimulation (Merzenich and White 1977; van den Honert and Stypulkowski 1987; Kral et al. 1998; Rebscher et al. 2001; Bierer and Middlebrooks 2002; Snyder et al. 2004).

An obvious methodological difference is that our BIC measurements specifically represent the activity of binaurally sensitive neurons, whereas previous studies measured activation patterns elicited by monaural stimulation. These studies typically compared widths of excitation between different electrode configurations at a fixed level above threshold (e.g., $6 \mathrm{~dB}$ ). When analyzed in this way, our monaural IC data also show broader activation patterns with monopolar stimulation than with bipolar stimulation. However, when spatial width of excitation in IC is plotted against peak response amplitude (Fig. 10C), both electrode configurations show similar spreads of activity over the range where the peak amplitudes overlap. This observation suggests that the critical difference between our study and others is not the use of binaural stimulation versus monaural stimulation but rather the differing methods for setting stimulus levels in the two configurations. Rather than using a fixed level above threshold, we adjusted stimulus level for each electrode configuration so that the EABR amplitude would remain constant. Because EABR amplitude grows more steeply with intensity for monopolar stimulation than for bipolar stimulation (Fig. 3B), we effectively used lower stimulus levels re threshold in the monopolar condition. Similarly, recent psychophysical studies have shown that spatial patterns of forward masking in human implant subjects are comparable between bipolar and monopolar stimulations when loudness is balanced between configurations (Kwon and van den Honert 2006). It may be that stimulus configurations and intensities that produce very broad excitation, as often used in physiological experiments, would result in an uncomfortably loud percept and thus would be avoided in clinical implant settings. Another possible explanation for our relatively selective monopolar stimulation (in comparison to the results of Snyder et al. 2004) is that we pushed the intracochlear electrodes against the modiolus with cotton to minimize thresholds by decreasing the distance between the electrodes and the excitable neural tissue. More narrow spatial patterns of excitation for monopolar stimulation may be a direct result of this decrease in distance or alternatively could be related to the presence of space-filling cotton, which may have reduced shunting current exiting the scala tympani through the round window (Whiten 2006).

We commonly saw a sudden, rapid expansion of the width of excitation in the IC at higher intensities (>5 dB re threshold) with monopolar stimulation (Fig. 11B). This was not observed with bipolar stimulation, which maintained relatively good spatial tuning over the range of intensities tested. This difference may provide an explanation for psychophysical results in cochlear implant subjects showing that decreases in speech perception scores at high levels are more common with monopolar stimulation than with bipolar stimulation (Franck et al. 2003).

\section{Potential use in human subjects}

Methods that have been used to match interaural electrodes with bilateral cochlear implants include psychophysical tests of pitch matching (van Hoesel and Clark 1997; Long et al. 2003) and binaural fusion 
(Eddington 2005). These are subjective measurements that do not always predict interaural electrode pairings giving the best ITD JNDs (Long et al. 2003) and are impractical in pediatric subjects. Another potential method is the use of radiographic imaging to measure the exact location of each electrode (Kos et al. 2005).

Our results with evoked potentials and neural response patterns in the IC suggest that evoked potentials can effectively be used to find the relative position of bilaterally implanted intracochlear electrode arrays. The method based on BIC amplitude to find interaural electrode matches needs to be tested in adult humans where comparisons can be made with psychophysical methods. The existence of a BIC has already been reported in one bilateral implant subject (Pelizzone et al. 1990), so the method should be feasible in humans. In a study of normal-hearing newborns, the BIC waveform was detectable in over half of the babies tested with acoustic clicks (Furst et al. 2004), suggesting that the required binaural brainstem circuits are in place at birth and that BIC amplitude might also be useful in matching interaural electrodes in bilaterally implanted infants (as young as 9-12 months). This would assist in the assignment of frequency-electrode maps so that stimulation in each ear is tonotopically aligned, which potentially could improve the proper development of spatial hearing and binaural fusion of speech in congenitally deaf individuals.

\section{ACKNOWLEDGMENTS}

We thank Connie Miller for the surgical assistance, Ken Hancock for the computer support, and Steve Colburn and Don Eddington for the valuable comments on the manuscript. This work was supported by NIH Grants R01 DC005775 and P30 DC005209. Z.M. Smith was partially supported by NIH Grant T32 DC00038.

\section{REFERENCES}

Baskent D, Shannon RV. Speech recognition under conditions of frequency-place compression and expansion. J. Acoust. Soc. Am. 113:2064-2076, 2003.

Baskent D, Shannon RV. Frequency-place compression and expansion in cochlear implant listeners. J. Acoust. Soc. Am. 116:31303140, 2004.

Bierer JA, Middlebrooks JC. Auditory cortical images of cochlearimplant stimuli: dependence on electrode configuration. J. Neurophysiol. 87:478-492, 2002.

Black RC, Clark GM. Differential electrical excitation of the auditory nerve. J. Acoust. Soc. Am. 67:868-874, 1980.

Blauert J. Spatial Hearing: The Psychophysics of Human Sound Localization, Revised Edition. Cambridge, Mass., MIT Press, 1997.

Boudreau JC, Tsuchitani C. Binaural interaction in the cat superior olive S segment. J. Neurophysiol. 31:442-454, 1968.

CAird D, SONTHeimer D, KLinke R. Intra- and extracranially recorded auditory evoked potentials in the cat. I. Source location and binaural interaction. Electroencephalogr. Clin. Neurophysiol. 61:50-60, 1985.

Dorman MF, Loizou PC, Rainey D. Simulating the effect of cochlearimplant electrode insertion depth on speech understanding. J. Acoust. Soc. Am. 102:2993-2996, 1997.

Durlach NI, Colburn HS. Binaural phenomena. In: Carterette E and Friedman MP (eds) Handbook of Perception. New York, Academic, pp. 365-466, 1978.

EDdington DK. Changes in fusion and localization performance when transitioning from monolateral to bilateral listening. In: Conference on implantable auditory prosthesis. Pacific Grove, California, 2005.

Franck KH, Xu L, Pfingst BE. Effects of stimulus level on speech perception with cochlear prostheses. J. Assoc. Res. Otolaryngol. 4:49-59, 2003.

Furst M, Levine RA, McGaffigan PM. Click lateralization is related to the beta component of the dichotic brainstem auditory evoked potentials of human subjects. J. Acoust. Soc. Am. 78:1644-1651, 1985.

Furst M, Bresloff I, Levine RA, Merlob PL, Attias JJ. Interaural time coincidence detectors are present at birth: evidence from binaural interaction. Hear. Res. 187:63-72, 2004.

Gordon KA, Papsin BC, Harrison RV. Toward a battery of behavioral and objective measures to achieve optimal cochlear implant stimulation levels in children. Ear Hear. 25:447-463, 2004.

Guinan JJ, JR., Norris BE, Guinan SS. Single auditory units in the superior olivary complex. II: locations of unit categories and tonotopic organization. Int. J. Neurosci. 4:147-166, 1972.

Kos Mi, Boex C, Sigrist A, Guyot JP, Pelizzone M. Measurements of electrode position inside the cochlea for different cochlear implant systems. Acta Otolaryngol. 125:474-480, 2005.

Kral A, Hartmann R, Mortazavi D, Kuinke R. Spatial resolution of cochlear implants: the electrical field and excitation of auditory afferents. Hear Res. 121:11-28, 1998.

Kuwada S, Yin TC, Syka J, Buunen TJ, Wickesberg RE. Binaural interaction in low-frequency neurons in inferior colliculus of the cat. IV. Comparison of monaural and binaural response properties. J. Neurophysiol. 51:1306-1325, 1984.

KWON BJ, van DEN HonerT C. Effect of electrode configuration on psychophysical forward masking in cochlear implant listeners. J. Acoust. Soc. Am. 119:2994-3002, 2006.

Litovsky R, Parkinson A, Arcaroli J, Sammeth C. Simultaneous bilateral cochlear implantation in adults: a multicenter clinical study. Ear Hear. 27:714-731, 2006.

Long CJ, Eddington DK, Colburn HS, Rabinowtiz WM. Binaural sensitivity as a function of interaural electrode position with a bilateral cochlear implant user. J. Acoust. Soc. Am. 114:1565-1574, 2003.

Long CJ, Deeks JM, Leow M-C, Carlyon RP. Simulations of bilateral cochlear implant speech reception in noise. In: Association for Research in Otolaryngology Midwinter Meeting. Daytona Beach, Florida, 2004.

Melcher JR. Cellular generators of the binaural difference potential in cat. Hear. Res. 95:144-160, 1996.

Merzenich MM, ReID MD. Representation of the cochlea within the inferior colliculus of the cat. Brain Res. 77:397-415, 1974.

Merzenich MM, White MW. Cochlear implants: the interface problem. In: Hambrecht FT and Reswick JB (eds) Functional Electrical Stimulation: Applications in Neural Prostheses. New York, Dekker, pp. 321-340, 1977.

Middlebrooks JC, Bierer JA. Auditory cortical images of cochlearimplant stimuli: coding of stimulus channel and current level. J. Neurophysiol. 87:493-507, 2002.

Nuetzel J, Hafter ER. Discrimination of interaural delays in complex waveforms. J. Acoust. Soc. Am. 69:1112-1118, 1981.

Pelizzone M, Kasper A, Montandon P. Binaural interaction in a cochlear implant patient. Hear. Res. 48:287-290, 1990. 
Pfingst BE, Xu L. Across-site variation in detection thresholds and maximum comfortable loudness levels for cochlear implants. J. Assoc. Res. Otolaryngol. 5:11-24, 2004.

QIU A, Schreiner CE, Escabi MA. Gabor analysis of auditory midbrain receptive fields: spectro-temporal and binaural composition. J. Neurophysiol. 90:456-476, 2003.

Rebscher SJ, Snyder RL, Leake PA. The effect of electrode configuration and duration of deafness on threshold and selectivity of responses to intracochlear electrical stimulation. J. Acoust. Soc. Am. 109:2035-2048, 2001.

Riedel H, Kollmeier B. Auditory brain stem responses evoked by lateralized clicks: is lateralization extracted in the human brain stem? Hear. Res. 163:12-26, 2002.

Rosen S, Faulkner A, Wilkinson L. Adaptation by normal listeners to upward spectral shifts of speech: implications for cochlear implants. J. Acoust. Soc. Am. 106:3629-3636, 1999.

Schon F, Muller J, Helms J. Speech reception thresholds obtained in a symmetrical four-loudspeaker arrangement from bilateral users of MED-EL cochlear implants. Otol. Neurotol. 23:710714, 2002.

Shannon RV, Zeng FG, Wygonski J. Speech recognition with altered spectral distribution of envelope cues. J. Acoust. Soc. Am. 104:2467-2476, 1998.

Siciliano C, Faulkner A, Rosen S, Saul L. Perceptual adaptation to a binaurally-mismatched frequency-to-place map. In: Association for Research in Otolaryngology Midwinter Meeting. Baltimore, Maryland, 2006.

Snyder RL, Rebscher SJ, Cao KL, Leake PA, Kelly K. Chronic intra- cochlear electrical stimulation in the neonatally deafened cat. I: expansion of central representation. Hear. Res. 50:7-33, 1990.

Snyder RL, Bierer JA, Middlebrooks JC. Topographic spread of inferior colliculus activation in response to acoustic and intracochlear electric stimulation. J. Assoc. Res. Otolaryngol. 5:305322, 2004.

Sontheimer D, CAird D, Kuinke R. Intra- and extracranially recorded auditory evoked potentials in the cat. II. Effects of interaural time and intensity differences. Electroencephalogr. Clin. Neurophysiol. 61:539-547, 1985.

van den Honert C, STypulkowski PH. Single fiber mapping of spatial excitation patterns in the electrically stimulated auditory nerve. Hear. Res. 29:195-206, 1987.

van Hoesel RJ, Clark GM. Psychophysical studies with two binaural cochlear implant subjects. J. Acoust. Soc. Am. 102:495-507, 1997.

Whiten DM. Electro-anatomical models of the cochlear implant. In: Harvard-MIT Division of Health Sciences and Technology. Cambridge, Massachusetts Institute of Technology, 2006.

Xu SA, Shepherd RK, Chen Y, Clark GM. Profound hearing loss in the cat following the single co-administration of kanamycin and ethacrynic acid. Hear. Res. 70:205-215, 1993.

YIN TC, CHAN JC. Interaural time sensitivity in medial superior olive of cat. J. Neurophysiol. 64:465-488, 1990.

ZUREK PM. Binaural advantages and directional effects in speech intelligibility. In: Studebaker GA and Hochberg I (eds) Acoustical Factors Affecting Hearing Aid Performance. Boston, Allyn and Bacon, pp. 255-276, 1993. 